\title{
Modern Electron Microscopy Applications In The Consumer Products Industry
}

\author{
H. Shen, D.S. Jacobs, J. L. Burns, Y.L. Boissy and D.W. Domaschko \\ Procter \& Gamble Co., Corporate Analytical Department, Cincinnati, Ohio 45252
}

Innovation in the consumer products industry is primarily driven by the desire to improve consumer satisfaction while reducing cost. The performance of consumer products, such as cleaning agents/detergents, beauty lotions/creams, paper products, toothpaste, and snacks and beverages, is closely related to their physical properties, which are largely influenced by the microstructure of finished products, raw materials, and processes. Electron microscopy is a critical technique for characterizing and evaluating microstructures. EM characterization therefore helps to drive the innovation of satisfactory consumer products.

The key EM techniques for us are low temperature direct-imaging transmission electron microscopy (cryo-TEM), freeze-fracture transmission electron microscopy (FF-TEM), and low temperature scanning electron microscopy (cryo-SEM) with X-ray microanalysis. Here we discuss the application of these techniques to several relevant systems.

\section{Dilute Aqueous Systems}

Cryo-TEM is often our choice of technique for dilute systems and excellent for examining systems in-use conditions. For detergents, the microstructures of surfactants at dilute wash conditions can impact cleaning performance. Interactions between various components such as salts, polymers and surfactants can induce significant changes in the microstructures, which lead to changes in performance in products such as toothpastes and hair shampoos. Nanoparticle morphology, for example the extent of clay platelet delamination, also directly affects the rheology and hence performance of many formulations. Figure 1 shows a few examples of how cryo-TEM provides insight into these systems.

\section{Concentrated and/or Viscous Systems}

Other products such as body lotions, hand creams, and lipsticks are too viscous to be examined by cryo-TEM. Their phase behavior and microstructures can be evaluated by FF-TEM or cryo-SEM. In general, FF-TEM can reveal fine details of microstructures such as lamellar or hexagonal liquid crystals because of its capability for high resolution. Cryo-SEM can provide additional information such as distribution of chemical ingredients and surface structure by including controlled etching and element microanalysis. A combination of FF-TEM and cryo-SEM can provide insights for the concentrated and viscous systems. The insights we obtain are used for problem solving in manufacturing processes, new product innovations, and performance enhancements. Examples in Figure 2 are used to illustrate some of these applications.

\section{Solid Systems}

Cryo-SEM, STEM, and TEM are applied to solid systems such as hair, skin, fabric, and polymers. Cryo-SEM along with microanalysis can be used to establish structure and function relationships for thin-film deposition or coatings on substrates such as hair, skin, and fabric. TEM thin sections can provide detailed information on hair dyeing, polymer films or fibers, and many others. A few examples of such studies are shown in Figure 3. 
Figure 1. Examples of microstructure studies in dilute liquid systems using cryo-TEM

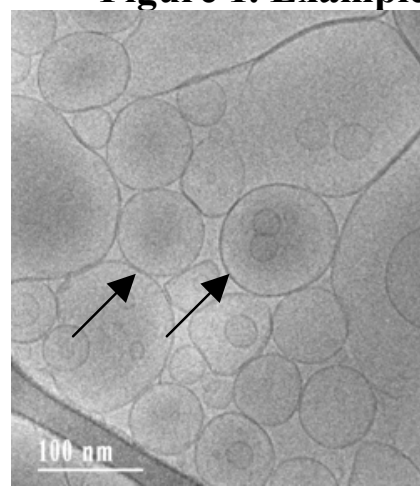

Presence of vesicles (arrows) in detergents may affect cleaning performance.

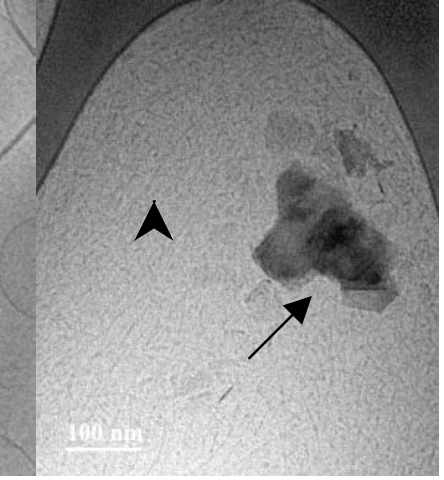

Presence of aggregates (arrow) and worm-like micelles (arrowhead) in solution may contribute to delivery of ingredients. softness

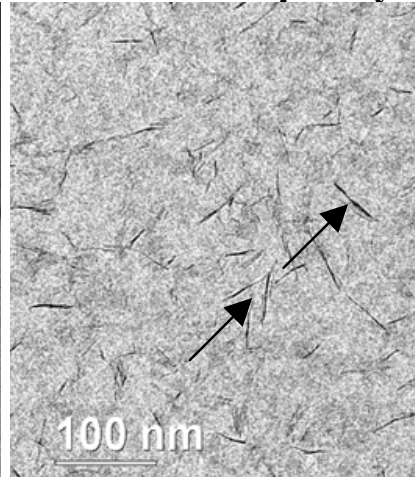

Delaminated clay platelets (arrows point to the edges) in softeners may enhance fabric

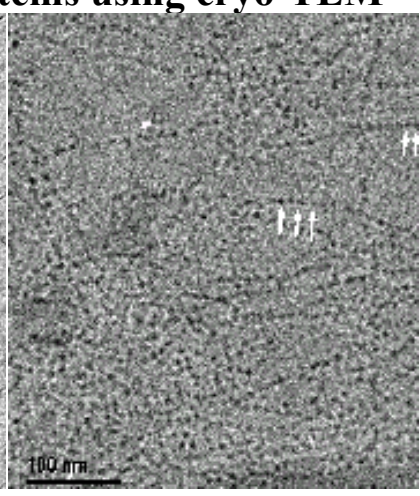

Spherical micelles attached to polymer chains (see arrows) are useful in some suspensions.

Figure 2. Examples of microstructure studies in concentrated and viscous systems

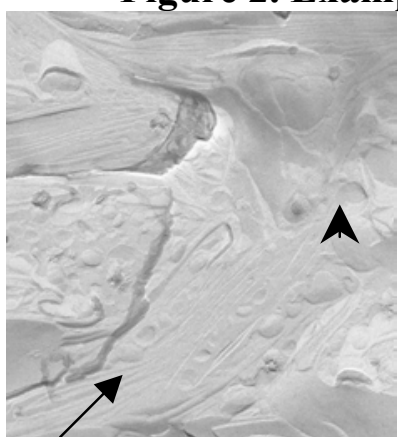

FF-TEM image of

vesicles (arrowhead) and lamellae (arrow) in cosmetic products. The structures may affect product texture/stability.

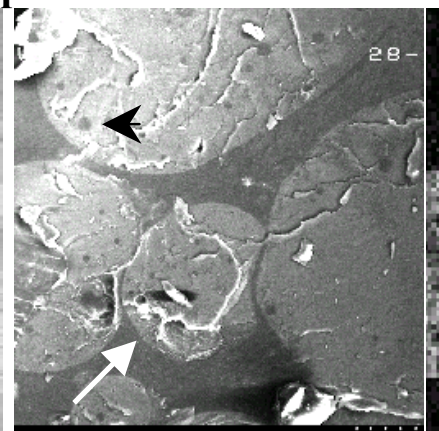

Cryo-SEM of W/O/W emulsion used in liquid detergents. Arrow-an oil droplet in water; arrowhead - a water drop in an oil droplet.

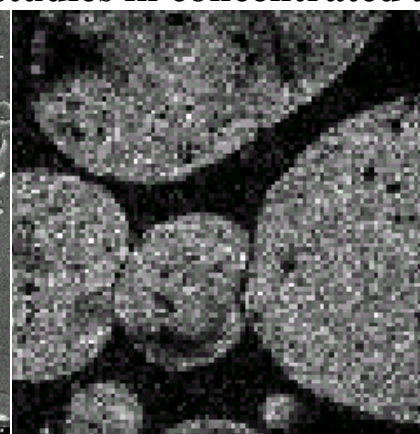

Silicon map of the same area on the left image. Images and map help show stability of emulsion.

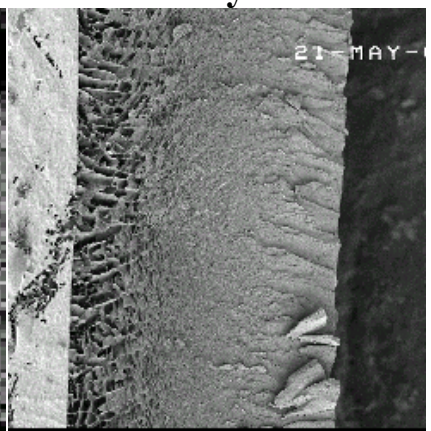

Cryo-SEM of an etched cross section of a film used in detergents. Holes may indicate water penetration. Left side was in contact with water.

\section{Figure 3. Examples of microstructure studies in solid-state systems}

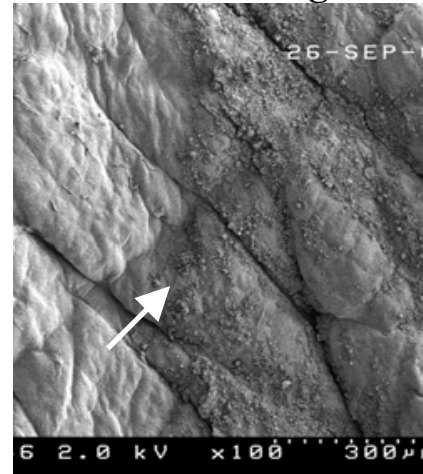

Cryo-SEM image of skin treated with body wash to deliver various benefits. Arrow indicates deposition.

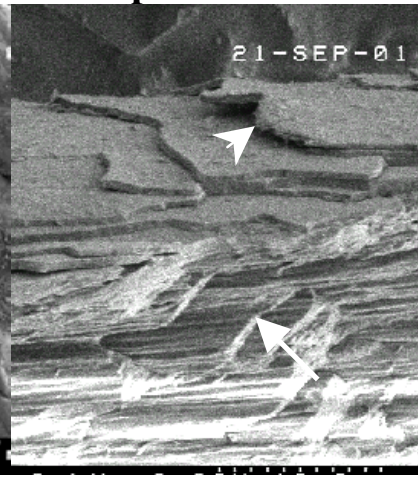

Cryo-SEM image of a partially fractured hair is used to study hairs. Arrowhead--lifted cuticle; various ingredients. arrow--fractured cortex.
Full scale $=250$ counts
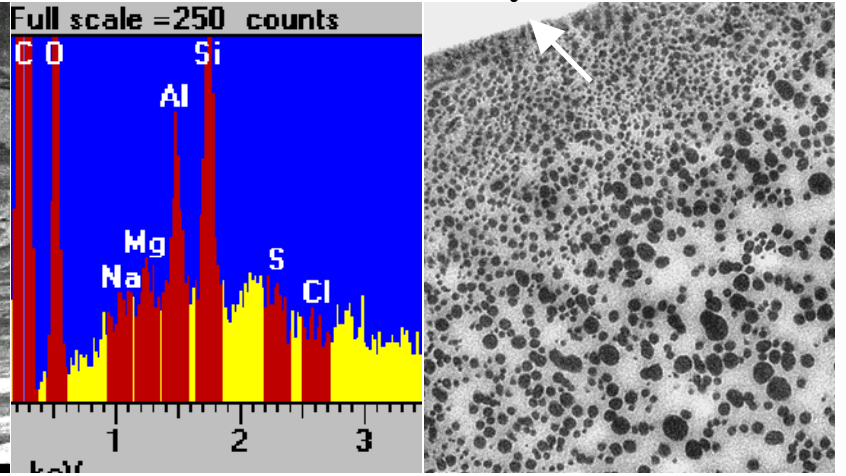

X-ray microanalysis of a TEM section of a polymer fiber is used to study the fiber used in paper deposition/penetration of products. Smaller discontinuous domains are near the surface (arrow). 